\title{
ON EXISTENCE, INCONSISTENCY, AND INDISPENSABILITY
}

\author{
HENRIQUE ANTUNES \\ Institute of Philosophy and the Humanities (IFCH), Unicamp, Campinas, BRAZIL \\ antunes.henrique@outlook.com
}

\begin{abstract}
In this paper I sketch some lines of response to Mark Colyvan's (2008) indispensability arguments for the existence of inconsistent objects, being mainly concerned with the indispensability of inconsistent mathematical entities. My response will draw heavily on Jody Azzouni's (2004) deflationary nominalism.
\end{abstract}

Keywords: Indispensability argument $\bullet$ deflationary nominalism $\bullet$ inconsistent theories inconsistent objects $\bullet$ dialetheism $\bullet$ principle of non-contradiction

An indispensability argument is an argument to the effect that we ought to be committed to the existence of mathematical/theoretical entities, given that they are indispensable to our best scientific theories. Indispensability arguments have been fairly influential in contemporary debates in the philosophy of mathematics and (to lesser extend) in the philosophy of science. In (2008) Mark Colyvan advances indispensability arguments that are specifically concerned with inconsistent or contradictory theories and whose purported effect is to establish that we ought to be committed to the existence of inconsistent objects. ${ }^{1}$ In this paper I shall sketch some lines of response to Colyvan's arguments, being mainly concerned with the indispensability of inconsistent mathematical entities. My response will draw on a relatively recent nominalistic interpretation of mathematics put forward by Jody Azzouni (2004).

The paper is structured as follows. In Section 1 I discuss the traditional versions of the indispensability argument for the existence of mathematical and theoretical entities. In Section 2 I present Colyvan's indispensability arguments for the existence of inconsistent objects. In Section 3, the main section of the paper, I present Azzouni's deflationary nominalism and argue that it can be straightforwardly adapted to handle inconsistent applied mathematical theories. I also suggest there that Azzouni's nominalism can be made compatible with a metaphysical version of the principle of non-contradiction (PNC). Finally, in Section 4 I (tentatively) propose a particular Logic of Formal Inconsistency as a logical framework for regimenting inconsistent applied mathematical theories - a framework which, in addition, incorporates that version of PNC. 


\section{Indispensability Arguments}

According to Hartry Field, "an indispensability argument is an argument that we should believe a certain claim... because doing so is indispensable for certain purposes (which the argument then details)" (1989, p.14). In particular, when the purposes at issue are explanations, it tells us that we should believe a certain claim (e.g., a claim that certain entities exist) because doing so is indispensable to certain explanations. So, for example, one may come to believe that there are rats living in the basement because this best explains all the rat-like noises one hears every night and the tiny pieces of food spread throughout the kitchen's floor one finds every morning. Similarly, one may come to believe that there is a burglar in the neighborhood because this best explains why so many things had gone missing in the last couple of days.

In philosophical contexts, indispensability arguments have been proposed to support the existence of entities to which (unlike rats) we don't seem to have any means of direct access - e.g., observation. These are the theoretical objects posited by scientific theories, such as electrons and black holes; and the abstract objects posited by mathematical theories, such as numbers and functions. Accordingly, there are two general versions of the indispensability argument corresponding to each of these two kinds of entity: the first one has been proposed as an argument for scientific realism, the thesis that theoretical entities exist; and the second, as an argument for mathematical realism (or Platonism), the thesis that mathematical entities exist and, furthermore, are mind- and language independent.

The indispensability argument for scientific realism purports to entail that we ought to be ontologically committed to theoretical entities given that they are indispensable to our current best scientific theories:

(1.1) Theoretical statements are indispensable to our current best scientific theories.

(1.2) We ought to believe in (at least some of) our best scientific theories.

(1.3) In particular, we ought to believe in the theoretical statements involved in our best scientific theories.

(1.4) Those statements contain existential quantifications over theoretical entities.

(1.5) The existential quantifier (and its natural language cognates) is an ontologically committing expression.

Therefore, we ought to have ontological commitment to theoretical entities.

Similarly, the indispensability argument for mathematical realism purports to entail that we ought to be ontologically committed to mathematical entities given that they are indispensable to our current best scientific theories: 
(2)

(2.1) Mathematical statements (pure or impure) are indispensable to our current best scientific theories.

(2.2) We ought to believe in (at least some of) our best scientific theories.

(2.3) In particular, we ought to believe in the mathematical statements involved in our best scientific theories.

(2.4) Those statements contain existential quantifications over mathematical entities.

(2.5) The existential quantifier (and its natural language cognates) is an ontologically committing expression.

Therefore, we ought to have ontological commitment to mathematical entities.

Some brief comments on each premise of both arguments are in order: premises (1.1) and (2.1) express that theoretical/mathematical statements cannot be eliminated from our current best scientific theories. This means that there are no alternatives to these theories which (i) are at least as attractive as their originals (according to some global theoretical virtues - e.g., boldness, familiarity, fecundity, scope, simplicity, success under testing) and (ii) are such that no theoretical/mathematical statement is involved in their formulations.

Premises (1.2) and (2.2) derive from the doctrine of naturalism; in a nutshell, the view that science, and science alone, is supposed to guide us in our beliefs about the world. Notice that (1.2) and (2.2) have normative force and that this force is transferred to the conclusions of the arguments. Premises (1.3) and (2.3) follow respectively from (1.2) and (2.2) and the doctrine of confimational holism, which asserts that no scientific hypothesis can be confirmed or disconfirmed in isolation, but only as part of a whole theory. In particular, it implies that any confirmation/disconfirmation garnered by a theory is also garnered by its theoretical and mathematical portions alike. $^{2}$

Premises (1.4) and (2.4) are pretty much uncontroversial. No one would reasonably deny that our current best scientific theories do contain prima facie existential quantifications over theoretical entities, such as atoms, electrons, black holes and the like; and over mathematical entities, such as functions, sets, numbers and the like. Premises (1.5) and (2.5) are nothing but Quine's criterion of ontological commitment; roughly, the doctrine that the first-order existential quantifier - and is its natural languages cognates, such as 'exists' and 'there is' - is the only ontologically committing expression of our and alien languages. According to that criterion, one should look for theorems of the form ' $\exists x(\ldots x \ldots)$ ' in order to identify those entities whose existence is assumed by a given theory.

Each of these premises has been challenged by anti-realists in the philosophy of science and in the philosophy of mathematics. To give a few examples concerning argument (2), premise (2.1) has been rejected by Hartry Field (1980), who attempts to 
show that mathematical statements are in fact dispensable to our best scientific theories by embarking on a program of nominalization of physical theory. The rejection of premise (2.2) is an immediate consequence of scientific instrumentalism, the view that although our scientific theories are extremely useful in applications, we should refrain from believing them - and, for that matter, from calling them 'true'. (2.3) is the main target of Balaguer's (1996) fictionalism, which maintains that although the nominalistic content of a scientific theory is to be taken as true, its mathematical content is to viewed as literally false (but theoretically useful). Although (1.4) and (2.4) are uncontroversial, it might be argued that the existential quantifications over theoretical/mathematical entities in our current best scientific theories are not to be taken at face, value and that an appropriate semantic analysis will reveal that they have no counterparts in the deep structure of scientific discourse. Premises (2.5) has been challenged by Jody Azzouni (2004), who endorses premises (2.1)-(2.4) but denies that the first-order existential quantifier (and its natural language cognates) is an ontologically committing expression. ${ }^{3}$

It is worth noticing that one may consistently subscribe to one of the two indispensability arguments above without subscribing to the other - or even subscribe to a version of one argument that is specifically concerned with a particular theory while rejecting similar versions concerning other theories. ${ }^{4}$ Of course, if one denies the existence of theoretical entities or of mathematical entities in general (or if one rejects the existence of the entities posited by a particular theory) then she has the burden of giving a sound response to the corresponding version of the indispensability argument. This miscellaneous strategy will be pursued in the following pages when dealing with Colyvan's indispensability arguments for the existence of inconsistent objects.

\section{Colyvan's Indispensability Arguments}

In (2008) Mark Colyvan formulates versions of the indispensability argument for the existence of inconsistent entities ${ }^{5}$. His alleged objective in the paper is not to positively argue for belief in inconsistent objects, thought, but rather to point out that the presence of inconsistent theories along the history of science poses a serious problem to scientific realism, since according to those arguments scientists committed to the truth of their inconsistent theories ought to have been ontologically committed to contradictory objects.

Colyvan (p.116) distinguishes three ways in which a scientific theory might end up inconsistent. According to him, a theory might be inadvertently inconsistent, in the sense that some of its assumptions unexpectedly conflict with other assumptions of the same theory or with assumptions of other well-established theories. It might 
be explicitly inconsistent, in the sense that it is an overtly inconsistent theory by itself (or overtly inconsistent with other well-established theories). Or it might be inconsistent because the theory invokes inconsistent mathematical theories in its very formulation. He (pp.116-9) goes on to elaborate on this distinction by presenting the following three examples of inconsistent theories:

(3) As an example of an inadvertently inconsistent theory, Colyvan mentions Newtonian gravitational theory. According to this theory, spiral galaxies have different rotations depending on their distribution of mass. If a galaxy is very massive and has a great concentration of mass away from its center, the theory tells us that gravity will hold it together causing the galaxy to spin like a wheel. On the other hand, if a galaxy has a high concentration of mass in its center, the center will spin faster than the spiral arms. However, in 1959 a galaxy was discovered which doesn't exhibit the kind of rotation that Newtonian gravitational theory predicts for it. This galaxy, called Triangulum Galaxy (M33), spins like wheel, even though its mass is highly concentrated in its center. ${ }^{6}$ Colyvan observes that this kind of conflict between theory and experimental results are pretty common in science, but as the instruments involved in the relevant experiments presuppose a great deal of theory, cases like the spiral galaxy are best viewed as conflicts between two parts of the same theory or between two or more theories, which turn out to be jointly inconsistent.

(4) As an example of an explicitly inconsistent theory, Colyvan mentions the theory of waves in open oceans, which assumes that oceans have infinite depths. This theory, when coupled with standard sonar techniques, which assume that oceans have finite depths - or when coupled with current cosmological theories which tell us that the Earth has a more or less elliptical form with a finite diameter - implies an outright contradiction: oceans are both finitely and infinitely deep.

(5) Finally, as examples of theories that are inconsistent because they invoke inconsistent mathematical tools, Colyvan mentions the original versions of mechanics, gravitational theory, and electromagnetic theory. All these assumed the early calculus as the underling mathematical theory, which was straightforwardly inconsistent:

... infinitesimals were taken to be non-zero (by stipulation and by the fact that one needed to divide by them) and yet at other times they were taken to be equal to zero. Moreover, within one proof... both these contradictory properties were invoked. Newton and Leibniz also seemed to have contradictory interpretations of the infinitesimals. (Colyvan 2008, p.118)

(3) and (4) are arguments for the existence of inconsistent theoretical entities namely, spiral galaxies which spin and don't spin like a wheel and oceans which have and don't have infinite depths. (5) is an argument for the existence of inconsistent 
mathematical objects - the infinitesimals posited by the early calculus — and may be schematically presented along the lines of argument (2):

(5)

(5.1) The early calculus was indispensable to 18th century best scientific theories.

(5.2) 18th century scientists ought to have believed in their best scientific theories.

(5.3) In particular, they ought to have believed in the theorems of early calculus involved in those theories.

(5.4) Those theorems contained existential quantifications over inconsistent mathematical entities (infinitesimals).

(5.5) The existential quantifier (and its natural language cognates) is an ontologically committing expression.

Therefore, 18th century scientists ought to have been ontologically committed to inconsistent mathematical entities.

Although my main objective in this paper is to discuss how Azzouni's deflationary nominalism undermines indispensability arguments for the existence of inconsistent mathematical objects, I will also have something to say about arguments (3) and (4) above. As the assessment of these cases will also draw on some of the theses advanced by him, it will be better to postpone their discussion until his position has been explained.

In the next section I will first present Azzouni's nominalism, focusing on three of its fundamental theses. I will then argue that his position can be straightforwardly adapted to handle inconsistent applied mathematical theories. In fact, I will show that given some of Azzouni's assumptions - in particular, his endorsement of a deflationary conception of truth - it implies a weak version of dialetheism (the thesis that there are true contradictions), but that this does not mean that contradictory objects must be assumed.

\section{A Nominalistic Response}

\subsection{Azzouni's Deflationary Nominalism}

As observed in Section 1, Jody Azzouni (2004) objects to the indispensability argument (2) for the existence of mathematical entities by rejecting premise (2.5) - i.e., by rejecting Quine's criterion of ontological commitment — while endorsing (2.1)(2.4). Accordingly, although Azzouni maintains that mathematical statements are in fact indispensable to our current best scientific theories, and that we ought to view (some of) those theories (along with the mathematics involved in their formulations) as literally true, he denies that we ought thereby to be committed to the existence of mathematical objects: 
...I take true mathematical statements as literally true; I forgo attempts to show that such literally true mathematical statements are not indispensable to empirical science, and yet, nonetheless, I can describe mathematical terms as referring to nothing at all. Without Quine's criterion to corrupt them, existential statements are innocent of ontology (I'll temporarily call this "the separation of existential truth from ontology" or, for short, "the separation thesis"). (Azzouni 2004, pp.4-5)

The separation thesis lies at the heart of Azzouni's nominalism and in order to understand it it is necessary to go through three of the main theses advocated in Azzouni (2004): (1) the indispensability of the truth idiom to scientific practice, (2) Azzouni's rejection of Quine's criterion of ontological commitment, and (3) his proposal of an existence predicate as the proper vehicle of ontological commitment. In the following pages, I will discuss each one of these theses in turn.

\subsubsection{The indispensability of truth}

Azzouni (2004, chapters 1 and 2) relies on a deflationary conception of truth in order to argue for the truth of (some of) our current best scientific theories (along with the mathematics involved in their formulations) - that is, in order to argue for premises (2.2) and (2.3) of argument (2). That is the view that the predicate 'is true' is not used to report substantive relations of correspondence between language and reality, but rather for the intra-linguistic purpose of facilitating semantic ascent and descent. According to the deflationary conception, ascribing truth to a given sentence ' $A$ ' is just an indirect way of asserting ' $A$ ' itself; the use of the truth predicate is thus taken to be entirely governed by the following schema:

$a$ is true if and only if $A$

(where $a$ stands for a name of the sentence ' $A$ ').

The T-schema enables us to eliminate any occurrence of the truth idiom whenever we are acquainted with the sentence referred to by $a$. Instead of asserting "Paris is the capital of France' is true', for example, one can simply assert 'Paris is the capital of France', and instead of asserting 'Fermat's last theorem is true', a number theorist can explicitly assert the statement of the theorem: 'For every natural numbers $a, b, c>0$ and for every $n>2, a^{n}+b^{n} \neq c^{n}$. This does not mean, however, that every use the truth idiom can be so eliminated, since truth ascriptions can also take place in situations where we are unacquainted with the sentence which truth is being ascribed to. Suppose, for example, that I am a convict catholic and that I assent to everything my parish priest, Father McKenzie, says in his Sunday sermons. Suppose, furthermore, that half an hour before this Sunday's Mass I assert 'The first sentence pronounced by Father McKenzie in today's homily will be true'. It is clear that in this case I cannot 
use the T-schema in order to eliminate the occurrence of the truth idiom (not until I listen to Father MacKenzie's homily).

Another kind of ineliminable use of the truth predicate is when truth is ascribed to an infinite set of sentences: if I want to assent to two sentences, I can either assert them individually or assert their conjunction; but if I want to assent to all the theorems of Peano Arithmetic (PA), I cannot help but to say 'All the theorems of Peano Arithmetic are true'. In this case, I am unable use the T-schema to eliminate the truth idiom, for this would require exhibiting each of PA's infinitely many theorems. Both kinds of ineliminable truth ascription are referred to as blind truth ascriptions.

Now, Azzouni argues that since infinitely (but recursively) axiomatizable theories, such as PA, do occur in science, scientists have no choice but to use the truth predicate in order apply them - i.e., in order to draw consequences from them. Suppose, for example, that I want to express my commitment to a theorem ' $A$ ' of PA, given my commitment to PA's axioms. In that case, Azzouni (p.18) observes, I must say

PA is true. PA entails $A$. ' $A$ ' is true. Therefore, $A$ (by the T-schema)

because since PA is not finitely axiomatizable I cannot eliminate the truth idiom from that inference using the T-schema. According to Azzouni, when coupled with the (empirical) indispensability of mathematical and scientific theories, this fact shows that we ought to be committed to the truth of those theories, or at least to the truth of some of their implications.

Azzouni does not think, however, that the (empirical) indispensability of a theory is a sufficient condition for its truth, for an indispensable theory may well be treated instrumentally by its practitioners:

Some philosophers may be tempted to think that if a theory is empirically indispensable then one has no choice but to accept its truth... This is just wrong: A theory, even an empirically indispensable one, can be treated instrumentally... What's needed is an implicit response to a question we must answer in order for the the attribution of an instrumentalist attitude toward an empirically indispensable theory to be cogent: How does the difference in attitude toward sentences we take to be true and toward sentences we take instrumentally manifest itself? (pp.34-35)

So, Azzouni requires that in order for a theory $T$ to be viewed as being treated instrumentally (rather than literally true) it must satisfy a manifestation condition; that is, there must be a manifest difference in attitude (on the part of T's practitioners) toward $T$ and toward theories not taken instrumentally — otherwise we could never tell them apart. According to him, this condition comes down to some of T's consequences being placed in quarantine, which means that although some of the implications of $T$ are in fact regarded as true, its practitioners refrain from believing them all; they quarantine some of $T$ 's consequences as either false or truth-valueless. 
The admission that some scientific theories may be treated instrumentally b.y its practitioners might be though to be a huge concession to general instrumentalism in the philosophy of science. After all, it might be that all of our current scientific theories have some of their consequences placed in quarantine and, so, that all of current science is treated instrumentally. Azzouni does not think this to be the case, though, since he claims that some of our current theories, especially the fundamental ones (such as general relativity and quantum mechanics), do not seem to satisfy the manifestation condition. Those theories, Azzouni insists, have no consequences placed in quarantine, and so must be regarded as literally true. "Current science, he says (p.45), is still in the business of searching for laws that apply without reservations to the world".

\subsubsection{Rejection of Quine's criterion of ontological commitment}

Quine's criterion of ontological commitment proposes that in order to determine the entities presupposed by a given theory $T$ (i.e., the entities that must exist in order for $T$ to be true), one must first regiment it into an interpreted first-order language $L$ and then look for the existentially quantified consequences of the resulting formalized theory — that is, sentences of the form ' $\exists x A$ ', where ' $A$ ' is any formula with ' $x$ ' free. The criterion is thus supposed to apply directly to first-order theories and can only be used to determine the ontological commitments of a theory expressed in natural language in an indirect way. ${ }^{7}$

Now, Azzouni detects in Quine's writings two arguments in support of his criterion. The first one draws on the model-theoretic interpretation of the objectual existential quantifier. The argument runs as follows: A (Tarskian) semantics for a first-order language $L$, where the quantifiers are interpreted objectually, is given by specifying a domain $D$, along with appropriate interpretations of the non-logical symbols of $L$ over $D$. The domain $D$ must be a set or a class of objects. The truth of an existentially quantified sentence $' \exists x A$ ' is then defined as the satisfaction of the formula ' $A$ ' by an object in $D$. So, in order for ' $\exists x A$ ' to be true there must be something which satisfies ' $A$ '.

Azzouni (pp.54-55) dispatches this argument by observing that it implicitly presupposes that the existential quantifier of the language in which the semantics is couched, the meta-language, is itself an ontologically committing expression. Hence, what the model-theoretic argument establishes is, at best, the conditional claim that if the meta-language existential quantifier is ontologically committing then the objectlanguage existential quantifier is ontologically committing, as well. In sum, "One can't read ontological commitments from semantic conditions unless one has already smuggled into those semantic conditions the ontology one would like to read off" (Azzouni 2004, p.55). 
The second argument advanced by Quine in favor of his criterion purports to show that the criterion is in fact trivially motivated - and, so, Azzouni calls this second argument triviality thesis: it maintains that because ordinary uses of 'there is' in the vernacular carry ontological commitment and because that idiom is straightforwardly regimented as the objectual existential quantifier of first-order logic, ' $\exists$ ' inherits the ontological role played by its natural language cognate ${ }^{8}$.

Azzouni (p.53) points out, however, that the claim that the ordinary language 'there is' is an ontologically committing expression is a substantial thesis - one for which Quine has provided no argument at all. This view faces the challenge of accounting for many uses of 'there is' in the vernacular which do not seem to carry ontological commitment. The paradigmatic examples come from discourse about fiction, which abounds with sentences where that idiom occurs. Here are some of Azzouni's examples:

(M) There are fictional mice that talk

(S) There are fictional detectives who are admired by some real detectives

Although these sentences are apparently true, we do not seem to countenance to the existence of the fictional objects they are about — presumably, Mickey Mouse and Sherlock Holmes. So, the supporters of the triviality thesis either have to assume the existence of fictional objects (contrary to our intuitions) or come up with some cogent explanation of why sentences such as the above are not real counter-examples to the claim that the ordinary language 'there is' is ontologically committing.

Azzouni (pp.63-78) considers several strategies for handling these potential counter-examples, and after carefully analyzing each one in turn and finding all of them wanting, concludes:

... Quine has no argument — at least no argument deserving the name "trivial" - for his taking the first-order objectual quantifier to be the sole conveyer of ontological commitment (in regimented languages). (p.79)

\subsubsection{Criterion of existence}

With Quine's criterion (for what discourse commits us to) out of stage, Azzouni (p.79) proposes that the commitments of a discourse or theory should be evaluated in terms of an existence predicate ' $E$ ' crafted precisely to carry ontological commitment. This move would then enable us to regiment the sentence $S$ above as follows:

$\exists x(x$ is a fictional detective $\wedge \exists y(y$ is a detective $\wedge E(y) \wedge y$ admires $x))$

And since ' $\exists$ ' is no longer seen as an ontologically committing expression, a commitment to the truth of S would not automatically commits us the existence of Sherlock Holmes or any other fictional entity. 
Given the failure of Quine's arguments in favor of his criterion, this seems the right way to go. But what does it mean to have an existence predicate, instead of the objectual existential quantifier, carrying ontological commitment? Quine thought his criterion to be logically prior to any metaphysical arguments about what exists and the triviality thesis was meant to show precisely this. But now that that thesis no longer stands, Azzouni observes, we are left with no metaphysically neutral standpoint from where to evaluate our (and alien) commitments and, so, we are forced either to embrace metaphysical nihilism or to look to for a criterion for what exists. Only after we have settled on one such criterion we can assign any meaning to the existence predicate in order to adjudicate between those terms of our language to which ' $E$ ' truthfully applies from those to which it doesn't.

Of course, there are several candidate criteria for what exists - observationality, location in space-time, casual efficacy - and since mathematical entities are usually described by Platonists as being abstract each one of them would suffice to rule them out. Azzouni (pp.91-97) argues, however, that the question of which one of these criteria is the correct one is metaphysically indeterminate since they cannot be adjudicated among on purely philosophical grounds. Nevertheless, he claims that despite this negative result our community of speakers has collectively adopted ontological independence of our linguistic practices and psychological processes as a criterion of existence. That is, it is a sociological fact that we, as a community, take something to exist if and only if it is independent of our linguistic practices and psychological processes. But what exactly does that mean?

Azzouni does not give an explicit characterization of the notion ontological independence. Rather, he offers several examples of ontologically dependent entities: contents of dreams and hallucinations, fictional and mythological entities and, crucially, mathematicalia. Consider, for example, the case of dreams and hallucinations: if I dream about an elf, that elf is completely dependent on a psychological process of mine. Likewise, if I hallucinate about a giant walking tree, that creature is a product of a particular psychological state I find myself in - induced perhaps by the use of some kind of drug. Fictional and mythological entities are no different, except in that they are dependent on our linguistic practices, instead. When Tolkien was writing about elfs, dwarfs, hobbits and all other sorts of strange creatures inhabiting the Middle Earth, he was not required to square their properties with anything else. He was simply stipulating truths about those creatures while writing his novels. Since all these entities are ontologically dependent either on our linguistic practices (writing a novel) or on our psychological processes (dreaming and hallucinating), they do not satisfy the criterion of existence favored by Azzouni and, so, exist in no sense at all. ${ }^{9}$

What about mathematical objects? Are they ontologically dependent on our linguistic practices or on our psychological processes the way fictional and hallucinated 
entities are? Azzouni thinks so. More specifically, he believes that although mathematicalia are independent of our psychological processes, they do depend on our linguistic practices. To support this claim, he (pp.99-100) observes that his criterion of existence imposes some epistemic constraints on the processes by means of which we come to acquire knowledge about existent objects; namely, the reliability of those processes must be susceptible to a non-trivial explanation. Crudely and roughly put, this means that when explaining why a given epistemic process $P$ is reliable in yielding knowledge about existent objects $x$ s we are not supposed to simply say 'because $P$ tells us that $x$ s are so-and-so'. According to Azzouni, fictional and mathematical entities alike do not satisfy the non-triviality requirement because while writing a novel or adopting a new mathematical axiom we do not worry about why we are justified in attributing a particular property to certain fictional or mathematical entities; they are simply stipulated to have that property. As a result, Azzouni concludes, mathematical objects are not ontologically independent of us and, therefore, exist in no sense at all.

To sum up: Azzouni argues for premises (2.2) and (2.3) of the indispensability argument (2) by taking into account the ineliminable role played by the truth idiom in scientific practice. He (pp.29-30) also thinks that to date attempts to show that mathematics is dispensable to science have not succeeded in achieving their goals. So, he endorses premise (2.1), as well. Nevertheless, Azzouni rejects the (Quinean) view that empirical indispensability is a sufficient condition for truth, since some indispensable scientific theories may be recognized as being treated instrumentally by its practitioners - as long as they satisfy the manifestation condition, that is, as long as some of their consequences are placed in quarantine. This does not undermine (2.2) and (2.3), though, given that not all of our current theories are taken instrumentally; at least some of them are regarded as literally true. Although the indispensability of mathematics to our current best scientific theories is taken to entail the existence of mathematical objects by the indispensabilist, this move from truth to existence relies crucially on the endorsement of Quine's criterion of ontological commitment (or of a natural language version of it). As we just saw, Azzouni blocks this move by rejecting Quine's criterion (by rejecting premise (2.5)) and proposing that our commitments should be evaluated instead in terms of an existence predicate ' $E$ ' interpreted in terms of the notion of ontological independence.

\subsection{Colyvan's Indispensability Arguments Revisited}

Now it is time to reconsider Colyvan's indispensability arguments for the existence of inconsistent of objects under the light of the theses presented above. I will first take up argument (4), which I think will be more easily dispatched, and then consider arguments (5) and (3) in turn. While discussing argument (5) I will address the 
question of what is the relationship between Azzouni's nominalism and dialetheism, the thesis that there are true contradictions.

Recall that argument (4) purported to show that we ought to be ontologically committed to contradictory theoretical objects - namely, oceans which have and do not have infinite depths - given that their existence is a consequence of the theory of waves in open oceans (when taken together with other well-established scientific theories). Now, it seems pretty clear that that (empirically indispensable) theory satisfies the manifestation condition for instrumentality: no scientist applying this theory would accept all of its consequences, but only those concerning the behavior of waves. In particular, no scientist would truly believe that the Earth has and does not have a more or less elliptical form or (to give a more mundane example) that a submarine navigating in the Atlantic could dive deeper and deeper without ever reaching the "bottom". Hence, some of the consequences of the theory are most likely seen as being placed in quarantine by its practitioners and, so, that theory is not taken to be literally true. Since one of the premises of argument (4) fails, the argument no longer stands.

As the reader might have already anticipated, a response to argument (5) is also straightforward: let's first assume, for the sake of argument, that those 18th century indispensable theories (mechanics, gravitational theory and electromagnetic theory) were taken to be true by its practitioners - i.e., let's grant premises (5.1)-(5.3). Now, given Azzouni's rejection of Quine's criterion of ontological commitment, this does not entail that 18th century scientists ought to have been committed to the mathematical entities quantified over by those theories - in particular, it does not entail that they ought to have been committed to inconsistent infinitesimals. As a matter of fact, given Azzouni's denial of the existence of mathematical entities, it follows that they ought not to have been committed to any mathematical object whatsoever.

It is worth noticing that this line of reasoning can be straightforwardly adapted to any inconsistent applied mathematical theory. Even if the relevant theory, despite being inconsistent, is taken to be true, we ought not to be committed to its inconsistent mathematical posits; and this is for the simple reason that we ought not to be committed to any mathematical objects whatsoever. According to Azzouni, consistent and inconsistent mathematical objects alike are not ontologically independent of our linguistic practices and, therefore, exist in no sense at all.

But what does Azzouni actually think about true inconsistent theories? Does he have any qualms about calling an inconsistent scientific theory 'true'? Apparently not. While responding to the instrumentalist argument that because scientists sometimes apply incompatible theories to the same phenomena those theories are not regarded as literally true, he writes:

[T] he considerations about blind truth ascriptions show that even if scientists were committed to contradictory theories, one could not blithely gloss this 
as a matter of the theories not being taken as true. That would be to mistake why the truth idiom is involved in scientific practice to begin with. The right move - if a philosopher insists on the collective inconsistency of scientific doctrine - is to focus on the logic used in science, to argue that scientists are using one or another paraconsistent logic, for example, instead of a classical one.

As I've already said, I prefer to leave the logic alone and take a closer look at how the so-called competing theories are justified in applications, in particular, to locate what more general theory they're being culled from, and in which ways they're differing approximations of that theory. Of course, I'm making an empirical claims here: It could turn out that scientists really are committed to inconsistent theories... (p.44)

So, although Azzouni prefers to attempt to analyze incompatible scientific theories as alternative idealizations deriving from a more fundamental one, he admits that if scientists were committed to inconsistent theories then they ought to be described as taking those theories as true. This is because according to the deflationary conception of truth calling a statement ' $A$ ' 'true' amounts merely to expressing a commitment to ' $A$ '.

In fact, it has been argued (by Beall and Armour-Garb (2003) and by Priest (2000)) that due to the semantic paradoxes the deflationary conception of truth leads directly to dialetheism. In Beall and Armour-Garb (2003), for example, the authors argue that because deflationism maintains that there is nothing more to the notion of truth than what is expressed by the T-schema there can be no principled delimitation of the range of sentences to which that schema applies in order to rule out its paradoxical instances; any well-formed sentence of our language will yield a corresponding bona fide instance of the T-schema, including the Liar sentence:

$\mathrm{L}$ is not true

And since that instance (i.e., $\mathrm{L}$ is true iff $\mathrm{L}$ ) leads directly to

$\mathrm{L}$ is true iff $\mathrm{L}$ is not true

it entails

$\mathrm{L}$ is true and $\mathrm{L}$ is not true

Hence, if Beall and Armour-Garb are right (as I think they are), Azzouni's endorsement of deflationism commits him to dialetheism.

I am, of course, piggybacking on Beall and Amour-Garb's argument for the claim that deflationism entails dialetheism, and if their argument turns out to be flawed I should then review my claim that Azzouni's endorsement of a deflationary conception 
of truth commits him to dialetheism. Although I won't rehearse the entire argument of Beall and Armour-Garb (2003), let me say a little bit more about it in order to attempt to convince the reader of its plausibility. The main idea of the argument is to point out that since deflationists hold that the truth predicate is not to be understood as reporting substantial relations between language and reality, there is nothing about the notion of truth that they can appeal to in order to place principled delimitations to its range. The predicate 'is true' is applicable to whatever sentence of our language, as long as it is well-formed. And since deflationists also maintain that uses of 'is true' are completely governed by the instances of the T-schema, this also means that there can be no principled restrictions to that schema either. ${ }^{10}$

The situation is different with respect to non-deflationist theories of truth, for in maintaining that the truth predicate expresses a substantive relation between language and reality, those theories can invoke one or another aspect of this relation in order to motivate a restriction in the range of the T-schema. Consider, for example, a simple version of the correspondence theory of truth, according to which a sentence is true iff it corresponds to a state of affairs that obtains. This theory has enough resources to limit the range of the T-schema because it entails that the truth predicate is inapplicable to sentences that do not correspond to a state of affairs. Hence, if a philosopher endorsing such a theory manages to successfully argue that no paradoxical sentence, such as $\mathrm{L}$ above, ever corresponds to a state of affairs he will then be capable of avoiding the paradox by denying that ' $\mathrm{L}$ is true iff $\mathrm{L}$ ' is an appropriate instante of the T-schema. No such route seems open to a deflationist (or so Beall and Armour-Garb's argument purports to show). ${ }^{11}$

It is worth emphasizing, however, that the version of dialetheism at stake is very weak, indeed. For consider the following semantic and metaphysical formulations of the principle of non-contradiction:

No sentence is simultaneously true and false

(PNC-M)

There are no contradictory objects

Let's call the denial of PNC-S semantic dialetheism and the denial of PNC-M metaphysical dialetheism. ${ }^{12}$ Now, just like Azzouni's rejection of Quine's criterion of ontological commitment blocks the move from the truth of mathematical theories to the existence of the purported objects those theories are about - i.e, it blocks the move from semantic to metaphysical realism in the philosophy of mathematics - it also blocks the move from semantic to metaphysical dialetheism: once Quine's criterion is gone, the acceptance of the truth of contradictory statements no longer compels us to assume the existence of the purported contradictory objects those statements are presumably about. Moreover, given Azzouni's argument for the non-existence of mathematicalia, it also follows that, as far as inconsistent mathematical theories are 
concerned, we ought not to commit ourselves to the contradictory objects over which they existentially quantify - again, because mathematical objects exist in no sense at all.

Does this mean that no contradictory object exist? Not necessarily. For it may be that there are objects that, unlike the contents of hallucinations and dreams and unlike mathematical and fictional entities, are independent of our linguistic practices and psychological processes and which nonetheless turn out to be contradictory. As far as Azzouni's criterion of existence is concerned, these objects would then be taken to exist, despite being inconsistent. To rule out this possibility - and, so, to rule out metaphysical dialetheism - it would be necessary to impose a further constraint on Azzouni's criterion for what exists, namely, that consistency is a necessary condition on existence. This condition would then yield the following modified version of PNCM:

(PNC-EC)

Every existent object is consistent

I confess I do not know how exactly to motivate PNC-EC. Perhaps, it could be argued that just like it is a sociological fact that we, as a community, take something to exits if and only if it is independent of our linguistic practices and psychological processes, it is likewise a sociological fact that we take consistency to be a necessary condition on what exists. This sort of consideration could then be bolstered up by pointing out that most examples of purported inconsistent objects come from mathematics and fiction. I will not try pursue this matter any further here, though. Rather, what I want to do next is to tentatively propose a logical framework for regimenting inconsistent applied mathematical theories, by assuming PNC-EC. Before that, I will give a few, very general, indications on why I think example (4) need not commit us to the existence of inconsistent theoretical objects.

In my view, theories which imply consequences about existing objects which contradict experimental results are to be corrected or utterly rejected. In particular, in view of the contradictory consequences about spiral galaxies alluded earlier, Newtonian gravitational theory (or the theories underlying the instruments involved) is to be seen as an anomalous theory which calls for further elaboration. How else could a scientific theory be corrected/rejected if not by being refuted by empirical experiments? Of course, there is a long road between an anomaly being found in a given theory and the theory being rejected altogether (one may find, for instance, that the current instruments are not accurate, that the results were misinterpreted, that the calculations were wrong, that the theory needed non-ad hoc supplementary hypotheses etc.), but once all attempted solutions have proven to fail, there is no other way except to reject the theory as false. Surely, there must be some empirical criteria according to which a theory is taken to be refuted, otherwise science would 
be totally unconnected to the (empirical) reality, and I suspect that the presence of contradictions like the one involving spiral galaxies (that is, contradictions involving theoretical predictions and results delivered by experiments) is a very good candidate for one such criteria. ${ }^{13}$

\section{A Logical Framework}

Now that we have seen how Azzouni's nominalism can be adapted to provide a cogent interpretation of the ontology of inconsistent mathematical theories, I would like to tentatively propose a logical framework for regimenting such theories which incoporates a formalized version of PNC-EC.

First of all, it is well-known that in order to formalize a contradictory theory one cannot stick with classical logic, since any theory whose underlying logic is classical turns out to be trivial in the presence of a pair of contradictory theorems - i.e. any contradictory theory classically entails any formula whatsoever of its language. This is due to the fact that the principle of explosion (PE) $-A \rightarrow(\neg A \rightarrow B)-$ is classically valid. ${ }^{14}$ Accordingly, we need a logic in which PE does not hold in general; that is, we need a paraconsistent logic. ${ }^{15}$ This is not enough, though, for, as we shall see, it will prove useful to have a way to formally express the consistency of a single formula (rather than a whole system) within the object-language. Although not all paraconsistent logics have the technical resources to meet this requirement, there is a particular family of paraconsistent logics — developed within the Brazilian tradition on paraconsistency - that clearly do: the so-called Logics of Formal (In)Consistency (LFIs). One distinguishing feature of the LFIs is that they internalize the notion of consistency within the object-language by means of a new (unary) sentential operator $\circ$ (called 'consistency operator'): ${ }^{16}$ given a formula $A$, the formula $\circ A$ is to be read ' $A$ is consistent' and expresses that the principle of explosion holds for $A$. Thus, although the LFIs reject PE, as all other paraconsistent logics do, they countenance a restricted version of PE, called principle of gentle explosion (PGE) and formally expressed by $\circ A \rightarrow(A \rightarrow(\neg A \rightarrow B))$.

In this final section I will propose a particular LFI as a candidate logical theory for regimenting inconsistent applied mathematical theories. The logic, known as LFI1, was first proposed in Carnielli, Marcos and de Amo (2000), and although the formulation of LFI1 to be adopted here is based off the one in Carnielli, Marcos and de Amo (2000), there are significant differences between them.

First, and foremost, while the quantifiers of LFI1 are given a substitutional interpretation in Carnielli, Marcos and de Amo (2000) — and in other works as well (such as Carnielli and Coniglio (2016)) - they will be given an objectual interpretation, instead. This is for an important reason: Quine explicitly formulated his criterion of 
ontological commitment in terms of objectual quantifiers. Only these, he maintained, are ontologically relevant, and although substitutional quantifiers were taken by him to be ontologically neutral, he viewed the attempt to exploit them in order to eliminate ontological commitments as a way of avoiding the concern for the ontology of theories. ${ }^{17}$ Azzouni's rejection of Quine's criterion, we have seen, was supposed to be a rejection of that criterion as it was formulated by Quine. Hence, in order to be in accord with Azzouni's views and also avoid the Quinean charge that the logical framework below evades ontological concerns because it resorts to substitutional quantifiers, it is necessary that the quantifiers be interpreted objectually.

Second, in Carnielli, Marcos and de Amo (2000) LFI1 is provided with a threevalued semantics with $0, \frac{1}{2}$ and 1 as truth values - where 1 and $\frac{1}{2}$ are designated and $\frac{1}{2}$ is the value of contradictory sentences. The semantics below countenances only two truth values, 0 and 1 (corresponding to false and true, respectively), and interprets the semantic value of a formula as the set of its truth values: the semantic value of a formula $A$ may be $\{0\}$, in which case $A$ is false, and false only; it may be $\{0,1\}$, in which case it is both true and false; and it may be $\{1\}$, in which case it is true, and true only. Although this difference is technically immaterial, a semantic theory countenancing exactly two truth values and assigning a set containing both values to contradictions better represents contradictory sentences as those that are both true and false.

Third, the way the predicate symbols are interpreted here differs both from the way they are interpreted in Carnielli, Marcos and de Amo (2000) and from the way they are interpreted in Carnielli and Coniglio (2016). In Carnielli, Marcos and de Amo (2000) each $n$-ary predicate $P$ is interpreted as a function $I_{P}$ assigning one of the truth values from the set $\left\{0, \frac{1}{2}, 1\right\}$ to each $n$-tuple of elements of the domain. And in Carnielli and Coniglio (2016) $P$ is interpreted as an ordered triple $\left\langle P_{+}^{\mathfrak{A}}, P_{b}^{\mathfrak{A}}, P_{-}^{\mathfrak{A}}\right\rangle$, where $\mathfrak{A}$ is a first-order structure and $P_{+}^{\mathfrak{A}}, P_{b}^{\mathfrak{A}}, P_{-}^{\mathfrak{A}}$ are, respectively, the set of $n$-tuples that satisfy $P$, the set of $n$-tuples that both satisfy and do not satisfy $P$, and the set of $n$-tuples that do not satisfy $P$ (these are assumed to be pairwise disjoint and their union is assumed to be identical to the domain). Although the interpretation of the predicate symbols to be adopted below resembles this second approach, there is a noteworthy difference: the semantic value of $P$ will be defined as an ordered pair $\left\langle P_{+}^{\mathfrak{A}}, P_{-}^{\mathfrak{A}}\right\rangle$, where $P_{+}^{\mathfrak{A}}, P_{-}^{\mathfrak{A}}$ are no longer assumed to be exclusive. This means that an $n$ tuple of elements of the domain can belong to both $P_{+}^{\mathfrak{A}}$ and $P_{-}^{\mathfrak{A}}$, in which case $P$ is an inconsistent predicate. Again, though this difference is technically unimportant, this way of interpreting the predicates provides a more natural description of the truth conditions of contradictory atomic formulas. ${ }^{18}$

The logical vocabulary of LFI1 will be composed by the sentential connectives $\neg, \wedge, \vee, \rightarrow$ and the inconsistency operator $\bullet$, the punctuation symbols $($,$) , ,, the first-$ order variables from $\mathscr{V}=\left\{x_{i}: i \in N\right\}$, the quantifiers $\forall$ and $\exists$, and the identity 
predicate $=$. The non-logical vocabulary of a formal theory based on LFI1 can be completely characterized by means of its first-order signature, defined as an ordered pair $\Sigma=\langle\mathscr{C}, \mathscr{P}\rangle$, where $\mathscr{C}$ is the set of the individual constants of $\Sigma$ and $\mathscr{P}=\left\langle\mathscr{P}_{n}\right\rangle_{n \geq 1}$ is a family of disjoint sets such that (1) the elements of $P_{n}$ are the $n$-ary predicate letters of $\Sigma$, and $(2)=\in \mathscr{P}_{2}{ }^{19}$

Given a first-order signature $\Sigma$, the elements of $T(\Sigma)=\mathscr{V} \cup \mathscr{C}$ will be called $\Sigma$ terms, and the elements of $[\Sigma]=\mathscr{C} \cup \bigcup_{n \in N} \mathscr{P}_{n} \cup\{\forall, \exists\}, \Sigma$-parameters. The set $F(\Sigma)$ of $\Sigma$-formulas is defined as usual, except for the inclusion of the following additional clause: if $A \in F(\Sigma)$ then $\bullet A \in F(\Sigma){ }^{20}$ The connectives $\circ$ and $\leftrightarrow$ are defined in LFI1 by:

$$
\begin{gathered}
\circ A==_{d f} \neg \bullet A \\
A \leftrightarrow B==_{d f}(A \rightarrow B) \wedge(B \rightarrow A)
\end{gathered}
$$

Definition 1. Let $\Sigma=\langle\mathscr{C}, \mathscr{P}\rangle$ be a first-order signature. A $\Sigma$-structure is a function $\mathfrak{A}$ on $[\Sigma]$ satisfying the following conditions:

(1) $\mathfrak{A}(\forall)=\mathfrak{A}(\exists)$ is a non-empty set $|\mathfrak{A}|$ (called the domain of $\mathfrak{A}$ ).

(2) For each $c \in \mathscr{C}, c^{\mathfrak{A}} \in|\mathfrak{A}|$.

(3) For each $P \in \mathscr{P}_{n}, P^{\mathfrak{A}}$ is a pair $\left\langle P_{+}^{\mathfrak{A}}, P_{-}^{\mathfrak{A}}\right\rangle$ such that $P_{+}^{\mathfrak{A}} \cup P_{-}^{\mathfrak{A}}=|\mathfrak{A}|^{n}$.

(4) $=_{+}^{\mathfrak{A}}=\{\langle a, a\rangle: a \in|\mathfrak{A}|\}$.

A function $s$ from $\mathscr{V}$ to $|\mathfrak{A}|$ will be called an $\mathfrak{A}$-assignment. If $t \in \mathscr{V}$ the $s$-denotation of $t$ in $\mathfrak{A}$ (written $t^{\mathfrak{A}}[s]$ ) is $s(t)$; and if $t \in \mathscr{C}$ the $s$-denotation of $t$ in $\mathfrak{A}$ (also written $\left.t^{\mathfrak{A}}[s]\right)$ is $t^{\mathfrak{A}}$.

In clause (3) of the above definition, $P_{+}^{\mathfrak{A}}$ and $P_{-}^{\mathfrak{A}}$ are called the extension and the anti-extension of $P$, and designate, respectively, the set of $n$-tuples that satisfy and the set of $n$-tuples that do not satisfy $P$. The requirement that $P_{+}^{\mathfrak{A}}$ and $P_{-}^{\mathfrak{A}}$ be exhaustive amounts to the fact that LFI1 does not allow for truth value gaps (and, so, is not a paracomplete logic). As noticed above, there is, however, no similar requirement to the effect that they must be exclusive. As far as the definition goes, $P_{+}^{\mathfrak{A}}$ and $P_{-}^{\mathfrak{A}}$ may have a non-empty intersection, in which case $P$ would have contradictory instances. Clause (4) guarantees that $=$ is properly interpreted as an identity predicate. This does not mean that $=$ is never contradictory, though: although $\langle a, a\rangle \in=_{+}^{\mathfrak{A}}$, for every $a \in|\mathfrak{A}|$, it may be that $\langle b, b\rangle \in={ }_{-}^{\mathfrak{A}}$, for some $b \in|\mathfrak{A}|$.

Definition 2. Let $\mathfrak{A}$ be a $\Sigma$-structure, $s$ an $\mathfrak{A}$-assignment and $A, B \in F(\Sigma)$. The set of truth values of $A$ under $\mathfrak{A}$ and $s$ (written $A^{\mathfrak{A}}[s]$ ) is inductively defined as follows: 
(1) If $A$ is $P\left(t_{1}, \ldots, t_{n}\right)$ then

$1 \in A^{\mathfrak{A}}[s]$ iff $\left\langle t_{1}^{\mathfrak{A}}[s], \ldots, t_{n}^{\mathfrak{A}}[s]\right\rangle \in P_{+}^{\mathfrak{A}} ;$

$0 \in A^{\mathfrak{A}}[s]$ iff $\left\langle t_{1}^{\mathfrak{A}}[s], \ldots, t_{n}^{\mathfrak{A}}[s]\right\rangle \in P_{-}^{\mathfrak{A}}$

(3) If $A$ is $B \wedge C$ then

$1 \in A^{\mathfrak{A}}[s]$ iff $1 \in B^{\mathfrak{A}}[s]$ and $1 \in C^{\mathfrak{A}}[s]$;

$0 \in A^{\mathfrak{A}}[s]$ iff $0 \in B^{\mathfrak{A}}[s]$ or $0 \in C^{\mathfrak{A}}[s]$

(5) If $A$ is $\bullet B$ then

$1 \in A^{\mathfrak{A}}[s]$ iff $1 \in B^{\mathfrak{A}}[s]$ and $0 \in B^{\mathfrak{A}}[s]$;

$0 \in A^{\mathfrak{A}}[s]$ iff $1 \notin B^{\mathfrak{A}}[s]$ or $0 \notin B^{\mathfrak{A}}[s]$

(7) If $A$ is $\forall v B$ then

$1 \in A^{\mathfrak{A}}[s]$ iff $1 \in B^{\mathfrak{A}}[s(a / v)]$, for every $a \in|\mathfrak{A}|$;

$0 \in A^{\mathfrak{A}}[s]$ iff $0 \in B^{\mathfrak{A}}[s(a / v)]$, for some $a \in|\mathfrak{A}|$

(8) If $A$ is $\exists v A$ then

$1 \in A^{\mathfrak{A}}[s]$ iff $1 \in B^{\mathfrak{A}}[s(a / v)]$, for some $a \in|\mathfrak{A}|$;

$0 \in A^{\mathfrak{A}}[s]$ iff $0 \in B^{\mathfrak{A}}[s(a / v)]$, for every $a \in|\mathfrak{A}|$
(2) If $A$ is $\neg B$ then

$1 \in A^{\mathfrak{A}}[s]$ iff $0 \in B^{\mathfrak{A}}[s]$

$0 \in A^{\mathfrak{A}}[s]$ iff $1 \in B^{\mathfrak{A}}[s]$

(4) If $A$ is $B \vee C$ then

$1 \in A^{\mathfrak{A}}[s]$ iff $1 \in B^{\mathfrak{A}}[s]$ or $1 \in C^{\mathfrak{A}}[s]$;

$0 \in A^{\mathfrak{A}}[s]$ iff $0 \in B^{\mathfrak{A}}[s]$ and $0 \in C^{\mathfrak{A}}[s]$

(6) If $A$ is $B \rightarrow C$ then

$1 \in A^{\mathfrak{A}}[s]$ iff $1 \notin B^{\mathfrak{A}}[s]$ or $1 \in C^{\mathfrak{A}}[s]$; $0 \in A^{\mathfrak{A}}[s]$ iff $1 \in B^{\mathfrak{A}}[s]$ and $0 \in C^{\mathfrak{A}}[s]$

In clauses (7) and (8), $s_{v}^{a}$ denotes an assignment that differs from $s$ in at most the value of $v$. Notice that these clauses provide an objectual interpretation for the quantifiers of LFI1. Notice also that both negation and conjunction are given their standard interpretations in clauses (2) and (3): a negated formula is true (false) iff its negatum is false (true); a conjunction is true iff both conjuncts are true, and false iff at least one of them is false. Accordingly, if a formula $A$ is both true and false then its negation $\neg A$ is both true and false, in which case the conjunction $A \wedge \neg A$ is both true and false, as well. Thus, if $A$ is true and false (i.e., if it is a dialetheia) then $A \wedge \neg A$ is a true contradiction. It is a straightforward task to check that $A, \neg A=B$ does not hold in LFI1 and that $\bullet A \leftrightarrow(A \wedge \neg A)$ is a valid schema. ${ }^{21}$

Enough for the semantics of LFI1. Let's consider now a corresponding deductive system. Given a first-order signature $\Sigma$ and formulas $A, B \in F(\Sigma)$, let's call $A$ a generalization of $B$ iff for some $n \in N$ and $v_{1}, \ldots, v_{n} \in \mathscr{V}, A$ is $\forall v_{1} \ldots \forall v_{n} B$. The axioms of $\mathscr{D}_{\Sigma}$ are then the generalizations of all instances of the following schemas:

Sentential axioms:

(A1)

(A2)

(A3)

(A4)

(A5)

(A6)

(A7)

(A8)

$A \rightarrow(B \rightarrow A)$

$A \rightarrow(B \rightarrow(A \wedge B))$

$(A \wedge B) \rightarrow A$

$(A \wedge B) \rightarrow B$

$A \rightarrow(A \vee B)$

$B \rightarrow(A \vee B)$
$(A \rightarrow(B \rightarrow C)) \rightarrow((A \rightarrow B) \rightarrow(A \rightarrow C))$

$(A \rightarrow C) \rightarrow((B \rightarrow C) \rightarrow((A \vee B) \rightarrow C))$
First-order axioms:

(A16) $\forall v A \rightarrow A[t / v]$

(A17) $\quad A[t / v] \rightarrow \exists v A$

(A18) $\quad A \rightarrow \forall v A$

(A19) $\quad \exists v A \rightarrow A$

(A20) $\quad \forall v(A \rightarrow B) \rightarrow(\forall v A \rightarrow \forall v B)$

(A21) $\quad \forall v(A \rightarrow B) \rightarrow(\exists v A \rightarrow \exists v B)$

(A22) $\neg \forall v A \leftrightarrow \exists v \neg A$

(A23) $\quad \neg \exists v A \leftrightarrow \forall v \neg A$ 


\begin{tabular}{|c|c|c|c|}
\hline 9) & $A \vee \neg A$ & (A24) & $\bullet \forall v A \leftrightarrow(\exists v \bullet A$ \\
\hline A10) & $A \leftrightarrow \neg \neg A$ & (A25) & $\bullet \exists v A \leftrightarrow(\exists v \bullet A \wedge \forall v \neg A)$ \\
\hline (A1 & $\circ A \rightarrow(A \rightarrow(\neg A \rightarrow B))$ & & \\
\hline & $\bullet A \rightarrow(A \wedge \neg A)$ & & Identity axioms: \\
\hline A & $\bullet(A \wedge B) \leftrightarrow((\bullet A \wedge B) \vee(\bullet B$ & & \\
\hline (A1 & $\bullet(A \vee B) \leftrightarrow((\bullet A \wedge \neg B) \vee(\bullet B \wedge \neg A))$ & (A26) & $v=v$ \\
\hline (A15) & $\bullet(A \rightarrow B) \leftrightarrow(A \wedge \bullet B)$ & (A27) & $v_{1}=v_{2} \rightarrow\left(A\left[v_{1} / v_{2}\right] \rightarrow A\right)$ \\
\hline
\end{tabular}

In axioms A16 and A17 $t$ must be free for $v$ in $A$, and in axioms A17 and A18 $v$ must not be free in $A$. In axiom A27 $v_{1}$ must be free for $v_{2}$ in $A$. The system $\mathscr{D}_{\Sigma}$ has modus ponens (MP) as its sole inference rule.

The definitions of a derivation of $A \in F(\Sigma)$ from $\Gamma \subseteq F(\Sigma)$ in $\mathscr{D}_{\Sigma}$, theorem of $D_{\Sigma}$ and LFI1-first-order theory are the usual ones. I will use the notations $\Gamma \vdash A$ and $\vdash A$ to express that $A$ is a logical consequence of $\Gamma$ in $\mathscr{D}_{\Sigma}$ and that $A$ is a theorem of $\mathscr{D}_{\Sigma}$, respectively. By a standard Henkin-style strategy, it can be proved that $\mathscr{D}_{\Sigma}$ is sound and complete with respect to the class of all $\Sigma$-structures.

I should mention at this point that LFI1, as well as many other LFIs, is prima facie unsuited to serve as the logical basis for a naive theory of truth (i.e., a theory of truth countenancing an unrestricted version of the T-schema). For consider a theory $T$ based on LFI1 such that True $(\ulcorner A\urcorner) \leftrightarrow A$ is a theorem of $T$, for every sentence $A$ of $T$. Consider now the open formula $\neg \operatorname{True}(v)$. By the Diagonal Lemma (which is assumed to hold in $T^{22}$ ), there is a sentence $L$ such that $L \leftrightarrow \neg \operatorname{True}(\ulcorner L\urcorner)$, which leads directly to True $(\ulcorner L\urcorner) \leftrightarrow \neg \operatorname{True}(\ulcorner L\urcorner)$. By A9 and A3, True $(\ulcorner L\urcorner) \wedge \neg \operatorname{True}(\ulcorner L\urcorner)$. But since True $(\ulcorner L\urcorner)$ is not know to be consistent, this fact does not by itself entail the triviality of $T$. Unfortunately, $T$ is not capable of avoiding all versions of the Liar paradox, for it can be shown that it is in fact trivial by a slight modification of the reasoning above: by applying the Diagonal Lemma to $\circ \operatorname{True}(v) \wedge \neg \operatorname{True}(v)$ instead of $\neg \operatorname{True}(v)^{23}$. This phenomenon shows that $T$ is not immune to revenge versions of the Liar paradox. ${ }^{24}$ It does not represent, however, a problem for the present proposal, for LFI1 is here supposed to serve as a logical basis for regimenting empirical theories, not semantic ones.

In order to make use of the deductive system above to regiment an inconsistent applied mathematical theory - when this theory is interpreted along the lines proposed in Section 3 - it will be necessary to introduce a unary predicate $E$ to its non-logical vocabulary. The formula $E(t)$ is to be interpreted as expressing that the term $t$ denotes an existent object. Now, there seems to be (at least) one axiom governing the relations between $E$ and the consistency operator of LFI1:

$$
\forall v_{1} \ldots \forall v_{n}\left(E\left(v_{1}\right) \wedge \cdots \wedge E\left(v_{n}\right) \rightarrow \circ P\left(v_{1}, \ldots, v_{n}\right)\right)
$$

for every $P \in \mathscr{P}_{n}$.

Axiom EC asserts that an atomic formula which exclusively concerns existent objects must be consistent, and formally expresses an atomic-level version of PNC-EC: in 
the presence of PGE (axiom A11), it implies that no non-trivial theory can have a pair of contradictory theorems $P\left(t_{1}, \ldots, t_{n}\right)$ and $\neg P\left(t_{1}, \ldots, t_{n}\right)$, when the terms $t_{1}, \ldots, t_{2}$ are known to satisfy $E .{ }^{25}$ As we shall see, EC can be generalized to all formulas of the relevant language, given the resources provided by LFI1. Before addressing this issue, though, it is worth considering a particular consequence of axiom EC; namely, the general consistency of the existence predicate:

$$
\forall v \circ E(v)
$$

PP asserts that the existence predicate itself must be consistent, irrespective of which terms it applies to. ${ }^{26}$ It expresses the requirement that there must be a neat division among the denoting and non-denoting terms of a theory. The rationale for this requirement is the idea that a regimented theory must be absolutely clear about its existential commitments, which would not be the case if it were allowed to have contradictory theorems of the form $E(t)$ and $\neg E(t) .{ }^{27}$

Now, axiom EC cannot be straightforwardly generalized to all formulas of the language - atomic and non-atomic - since the quantifiers of LFI1 are supposed to range over all items in the domain, which comprises both existent and non-existent objects - i.e., it comprises objects which both belong and do not belong to the interpretation of $E$ in a given structure (see Definition 2, clauses (7) and (8)). This means that any formula $A$ in which a (n unrestricted) quantifier occurs will not exclusively concern existent objects, even if all terms occurring free in $A$ are known to satisfy $E$. Hence, we are not entitled to asserting the consistency of $A$, if we are to stick to a strict formulation of PNC-EC.

There is, however, a simple way by means of which this difficulty can be overcome: instead of formulating the generalized version of EC with respect to any formula $A$ whatsoever, it can be formulated with respect to the result of relativizing all quantifiers occurring in $A$ to the existence predicate. This process will then guarantee that, unlike the original formula $A$, the resulting formula, $A^{i}$, concerns no objects other than existing ones (that is, those satisfying the existence predicate). The exact formulation and proof of this generalized version of EC require two preliminary steps. The first one is to give a precise definition of $A^{i}$, for any formula $A$. This is a fairly simple task:

Definition 3. Let $\Sigma=\langle\mathscr{C}, \mathscr{P}\rangle$ be a first-order signature such that $E \in \mathscr{P}_{1}$. Let $i$ : $F(\Sigma) \longrightarrow F(\Sigma)$ be an interpretation function satisfying the following conditions:

(1) If $A$ is an atomic formula then $A^{i}=A$.

(2) If $A$ is $\# B$, with $\# \in\{\neg, \bullet\}$, then $A^{i}$ is $\# B^{i}$.

(3) If $A$ is $B \# C$, with $\# \in\{\wedge, \vee, \rightarrow\}$, then $A^{i}$ is $B^{i} \# C^{i}$. 
(4) If $A$ is $\forall v B$ then $A^{i}$ is $\forall v\left(E(v) \rightarrow B^{i}\right)$.

(5) If $A$ is $\exists v B$ then $A^{i}$ is $\exists v\left(E(v) \wedge B^{i}\right)$.

The second step is to notice that the consistency operator o propagates across all sentential connectives of LFI1 and across the universal quantifier; that is, the following schemas all hold in LFI1:

$$
\begin{aligned}
& \circ A \rightarrow \circ(\neg A) \quad(\circ A \wedge \circ B) \rightarrow \circ(A \wedge B) \quad(\circ A \wedge \circ B) \rightarrow \circ(A \rightarrow B) \\
& \circ A \rightarrow \circ(\bullet A) \quad(\circ A \wedge \circ B) \rightarrow \circ(A \vee B) \quad \forall x \circ A \rightarrow \circ \forall x A
\end{aligned}
$$

Together with EC, PP and Definition 3, these properties are all we need to prove the generalization of EC:

Theorem 4. Let $T$ be a $\Sigma$-theory having EC as one of its non-logical axioms. Let $A \in$ $F(\Sigma)$ and suppose that all terms free in it are among $t_{1}, \ldots, t_{n}$ (provided that none of $t_{1}, \ldots, t_{n}$ is bound in $\left.A\right)^{28}$. Then,

$$
T \vdash E\left(t_{1}\right) \wedge \cdots \wedge E\left(t_{n}\right) \rightarrow \circ A^{i}
$$

The proof of this theorem proceeds by a straightforward induction on the complexity of $A$. Corollary 5 below is an immediate consequence of Theorem 4, while Corollary 6 follows from that theorem and the principle of gentle explosion (A11):

Corollary 5. If $A \in F(\Sigma)$ is quantifier-free and $t_{1}, \ldots, t_{n}$ are all the terms occurring in A then $T \vdash E\left(t_{1}\right) \wedge \cdots \wedge E\left(t_{n}\right) \rightarrow \circ A$.

Corollary 6. Let $A \in T(\Sigma)$ be a sentence such that all individual constants occurring in A are among $c_{1}, \ldots, c_{n}$. If $T \vdash E\left(c_{i}\right)$, for every $1 \leq i \leq n$, and $T \vdash A^{i} \wedge \neg A^{i}$ then $T$ is a trivial theory; that is, $T \vdash B$, for all $B \in F(\Sigma)$.

I claim that Theorem 4 and corollaries 5 and 6 appropriately capture the content of PNC-EC in the formal context of LFI ${ }^{29}$. Each one of them, but specially Corollary 6 , expresses the requirement that although a given theory may well be contradictory, it is not supposed, on pain of triviality, to have factual contradictory theorems; that is, theorems which exclusively concern objects taken to exist by that very same theory. Accordingly, there is at least an initial plausibility to using LFI1, together with axiom EC, to regiment inconsistent applied mathematical theories - when these are interpreted along the lines of Section 3. Of course, this does not mean that the apparatus developed in the foregoing pages does actually work. The only way I know of putting it to the test is to try to regiment an inconsistent applied theory. This is, however, a huge task and I will not try undertake it here.

It is worth emphasizing that the formal formulation of PNC-EC within LFI1 by means of Theorem 4 was only made possible by the resources provided by that logic. 
Crucially, by the fact that although LFI1 (and all logics in the LFI-family) rejects the general validity of the principle of explosion, it does ratify a restricted version of explosion, codified in the principle of gentle explosion. This feature, which is not shared by many other paraconsistent logics, allowed us to sort out those formulas that satisfy the principle of non-contradiction, relating them to the existence predicate $E$.

\section{Summary}

In this paper I tried to develop some lines of response to Colyvan's indispensability arguments for the existence of inconsistent objects. Concerning inconsistent theoretical entities, I only gave some hints on why I think example (3) is not to be taken as an argument for the existence of inconsistent objects. Example (4) was accounted for by invoking Azzouni's manifestation condition in order to show that the theory of waves in open oceans is not regarded as literally true by its practitioners - and, so, that its empirical indispensability does not commit us to its contradictory posits. As for inconsistent mathematical entities, I tried to sketch an objection to argument (5) which was based on Azzouni's deflationary nominalism, a theory according to which mathematical objects exist in no sense at all. This proposal led me to investigate whether a particular logic of formal (in)consistency, the logic LFI1, could serve as an adequate framework for regimenting inconsistent applied mathematical theories when a metaphysical version of the principle of non-contradiction is adopted. LFI1 turned out to have some desirable properties which make it a good candidate for the purposes at hand, specially in view of Theorem 4 above.

\section{References}

Azzouni, J. 2004. Deflating Existential Consequence: A Case for Nominalism. Oxford University Press.

Balaguer, M. 1996. A Fictionalist Account of the Indispensability of Applications of Mathematics. Philosophical Studies 83: 291-314.

Beall, J. C.; Armour-Garb, B. 2003. Should Deflationists Be Dialetheists? Noûs 37: 303-324.

Burgess, J. P. 1983. Why I Am Not a Nominalist. Notre Dame Journal of Formal Logic 24: 93-105.

Carnielli, W. A.; Coniglio, M. E. 2016. Paraconsistent Logic: Consistency, Contradiction and Negation. Springer.

Carnielli, W. A.; Marcos, J.; De Amo, S. 2000. Formal Inconsistency and Evolutionary Databases. Logic and Logical Philosophy 8: 115-152.

Colyvan, M. 2001. The Indispensability of Mathematics. Oxford University Press.

- 2008. The Ontological Commitments of Inconsistent Theories. Philosophical Studies 141: $115-123$.

- 2008a. Who's Afraid of Inconsistent Mathematics?. ProtoSociology 25: 24-35. 
2009. Applying Inconsistent Mathematics. In: Ot'avio Bueno; Øystein Linnebo (eds.) New Waves in Philosophy of Mathematics, pp.160-172. Palgrave Macmilan.

Field, H. 1980. Science Without Numbers: The Defence of Nominalism. Princeton University Press.

- 1989. Realism, Mathematics and Modality. Blackwell.

Priest, G. 2000. Truth and Contradiction. The Philosophical Quarterly 50: 305-319.

2006. In Contradiction. 2nd ed. Oxford University Press.

Putnam, H. 1971. Philosophy of Logic. Harper \& Row.

Quine, W. V. O. 1969a. Epistemology Naturalized. In: Ontological Relativity and Other Essays, pp.69-90. Columbia University Press.

- 1969b. Existence and Quantification. In: Ontological Relativity and Other Essays, pp.91-113. Columbia University Press.

- 1990. Pursuit of Truth. Harvard University Press.

Resnik, M. 1995. Scientific vs. Mathematical Realism: The Indispensability Argument. Philosophia Mathematica 3: 166-174.

\section{Notes}

${ }^{1}$ Throughout this paper, I take 'inconsistent' and 'contradictory' to be synonymous. This need not be the case, though, since there are interpretations of these notions according to which they do not coincide (see Carnielli and Coniglio 2016, ch.1). The use of 'contradictory' and 'inconsistent' adopted here is in accord with the logical framework presented in Section 4, since in that logic the notions of contradictoriness and inconsistency are provably equivalent. 2 The doctrine of naturalism stems, of course, from Quine (1969a), who also supported confirmational holism and was one of the first philosophers to rely on indispensability considerations in order to justify the belief in mathematical objects. The indispensability argument for the existence of mathematical entities can be found in scattered passages from Quine's writings, and, as far as I know, was explicitly formulated for the first time by Putnam (1971). For this reason it came to be called the 'Quine-Putnam Indispensability Argument'.

${ }^{3}$ I will have a lot more to say about Azzouni's position in Section 3.

${ }^{4}$ In responding to argument (2), for example, reconstructive nominalists attempt to show that current science can dispense with mathematical entities without attempting to do the same for theoretical entities. Their reconstructive programs propose new versions of one or another scientific theory in such a way that although the resulting nominalized theories do not quantify over mathematical abstracta (or so they claim), they do quantify over theoretical entities. On the other hand, a philosopher may hold that the indispensability of mathematics to science shows mathematics to be true (and mathematical entities to exist), irrespective of whether the scientific theories in which they are applied are actually true (and, so, irrespective of whether the theoretical entities they assume to exist actually exist). This may be maintained on the grounds that mathematics should be regarded as true because of its inferential role in scientific practice (this view is advanced by Michael Resnik in (1995)). Finally, a philosopher may proceed in a piecemeal way, by considering each particular theory to see whether or not it commits us to the existence of the purported objects they are purportedly about. As we shall see, this strategy is adopted by Azzouni, who holds that some theories are, 
in fact, taken instrumentally by its practitioners, whereas others theories are taken as literally true.

${ }^{5}$ See also Colyvan (2008a) and (2009).

${ }^{6}$ Colyvan (p.116) notices that since the discovery of galaxy M33 other galaxies were observed which have the same "anomalous behavior".

${ }^{7}$ As Azzouni (p.50) points out, philosophers very often retain Quine's criterion of ontological commitment while abandoning the restriction to first-order theories. In fact, most of the times they do not even require that the relevant theory must be a formalized one, implicitly relying on a (n unstated) natural language version of Quine's criterion - this situation is testified, for example, in Colyvan's formulation the indispensability arguments above.

${ }^{8}$ See (Quine 1990, pp.26-27), for example.

${ }^{9}$ The above talk about entities that exist in no sense at all may strike the reader as a very awkward way of speaking. Worse, it seems to entagle us in the old problem of the denial of existence. After all, if Sherlock Holmes is an entity, he must somehow exist and, so, it is certainly not true that he exists in no sense at all. Talking about entities in this context, Azzouni (p.91) observes, is, however, just a way of avoiding the cumbersomeness of semantic ascent: instead of saying 'Sherlock Holmes' is a singular term that refers to nothing at all' or "Sherlock Holmes' is a singular term that refers to nothing at all, but nonetheless satisfies the predicate "is admired by many real detectives" it is clearly much more convenient to simply say 'Sherlock Holmes exists in no sense at all' and 'Sherlock Holmes exists in no sense at all, but he is admired by many real detectives'.

${ }^{10}$ Azzouni explicitly endorses the thesis of the universal applicability of the truth predicate:

... I claim that the truth predicate must be univocal - that is, that one and only one truth predicate is applicable to all the sentences of our language. Notice that this requirement arises for a really simple reason: any group of sentences, however heterogeneous, provided only that they're sentences of our language, may be asserted by someone (and, consequently, blindly ascribed to by someone else). That is, the univocality of truth doesn't depend of the sentences of our language being any more closely connected than that. (Azzouni 2004, p.27)

Notice, however, that instead of relying on purely theoretical considerations about deflationism, he maintains that since any sentence of our language is in principle susceptible of being blindly asserted, there can be no restrictions to the range of the truth predicate.

${ }^{11}$ One traditional attempt to respond to the paradox of the Liar while retaining all instances of the T-schema consists in rejecting the inference from (*) to $(* *)$ - that is, denying that the inference from $A$ iff not- $A$ to $A$ and not- $A$ is generally valid. One way of supporting this move is to maintain that some sentences (crucially, the paradoxical ones) lack a truth value. For if a sentence $A$ is neither true nor false then both $A$ and not- $A$ fail to be true, in which case $A$ and not- $A$ fail to be true, as well. On the other hand, $A$ iff not- $A$ may be regarded as true even if $A$ is valueless (this depends, of course, on they way the iff part is interpreted).

However, rejecting the inference from $A$ iff not- $A$ to $A$ and not- $A$ does not by itself suffice to block the inference from (*) to $(* *)$, for this requires showing in a non-ad-hoc way that $\mathrm{L}$ in fact lacks a truth value - after all, the inference from $A$ iff not- $A$ to $A$ and not- $A$ seems to go through if we add a premise stating that $A$ is either true or false. Moreover, the truth-value gap solutions to the Liar are known to be vulnerable to the so-called revenge paradoxes. One 
typical example of these is the following: consider the sentence $\mathrm{L}$ again. According to the gap-theorist, L may be true, false, or valueless. If L is true then it is not true (by (*)); and if it is either false or valueless then it is not true, in which case it is true (by (*) again). In both cases, $\mathrm{L}$ is both true and not true.

${ }^{12}$ By 'contradictory object' I mean an object satisfying a pair of contradictory predicates.

${ }^{13}$ As the brief considerations above bypass several thorny issues in the philosophy of science, I don't claim to have nearly proven my point, but only given (what I see as) a promising suggestion on how inadvertently inconsistent theories are to be understood. Of course, this idea needs much further elaboration.

${ }^{14}$ From now on, I will drop the convention of using single quotation marks for mentioning expressions.

15 A logic $L$ (whose language contains a negation operatior $\neg$ and whose consequence relation is denoted by $\vdash_{L}$ ) is paraconsistent iff it is not the case that $A, \neg A \vdash_{L} B$, for every formulas $A$ and $B$.

${ }^{16}$ As we shall see, some LFIs are formulated in a language containing an inconsistency operator $\bullet$ instead of $\circ$.

17 See Quine (1969b), pp.104-107.

18 The axiomatic system below also differs from the one in Carnielli, Marcos and de Amo (2000) in that it has modus ponens (MP) as its sole inference rule, whereas the system in Carnielli, Marcos and de Amo (2000) also contains inference rules for the quantifiers.

19 The sets $\mathscr{C}, \bigcup_{n \in N} \mathscr{P}_{n}, \mathscr{V} \cup\{(),,,, \neg, \bullet, \wedge, \vee, \rightarrow, \forall, \exists\}$ are assumed to be pairwise disjoint.

${ }^{20}$ Henceforth, I will adopt the usual conventions for the omission of parenthesis and assume the usual definitions of a bound (free) occurrence of a variable in a formula, a bound (free) variable of a formula, and a term free for a variable in a formula. I will also make use of the notation $A[t / u]$ for the result of substituting all free occurrences of $u$ in $A$ by $t$.

${ }^{21}$ An $\mathfrak{A}$-assignment $s$ is said to satisfy $A \in F(\Sigma)$ in $\mathfrak{A}\left(\mid=_{\mathfrak{A}} A[s]\right)$ iff $1 \in A^{\mathfrak{A}}[s]$, and $A$ is said to be true in $\mathfrak{A}$ (written $\models_{\mathfrak{A}} A$ ) iff $\models_{\mathfrak{A}} A[s]$, for every $\mathfrak{A}$-assignment $s$. A is a logical consequence of $\Gamma \subseteq F(\Sigma)$ (written $\Gamma \models A$ ) iff for every structure $\mathfrak{A}$ and every $\mathfrak{A}$-assignment $s,=_{\mathfrak{A}} A[s]$, if $=_{\mathfrak{A}} B[s]$, for every $B \in \Gamma$. Finally, $A$ is logically valid (written $\models A$ ) iff $\emptyset \models A$.

${ }^{22}$ In its simplest form, the Diagonal Lemma (for a given theory $T$ ) states that for every formula $A$ of $T$ with exactly one free variable, there is a sentence $D$ such that $T \vdash D \leftrightarrow A(\ulcorner D\urcorner)$.

${ }^{23}$ By the Diagonal Lemma, there is a sentence $L$ such that

$$
\text { (*) } T \vdash L \leftrightarrow(\circ \operatorname{Tr}(\ulcorner L\urcorner) \wedge \neg \operatorname{Tr}(\ulcorner L\urcorner))
$$

By A9, $\bullet \operatorname{Tr}(\ulcorner L\urcorner) \vee \circ \operatorname{Tr}(\ulcorner L\urcorner)$. Suppose that $\bullet \operatorname{Tr}(\ulcorner L\urcorner)$. By A12 and A4, $\operatorname{Tr}(\ulcorner L\urcorner)$, and, since, $T \vdash L \leftrightarrow \operatorname{Tr}(\ulcorner L\urcorner)$, we obtain $L$. Suppose now that $\circ \operatorname{Tr}(\ulcorner L\urcorner)$. By A9, $\operatorname{Tr}(\ulcorner L\urcorner) \vee \neg \operatorname{Tr}(\ulcorner L\urcorner)$. If $\operatorname{Tr}(\ulcorner L\urcorner)$, then $L$. If, on the other hand, $\neg \operatorname{Tr}(\ulcorner L\urcorner)$, then $\circ \operatorname{Tr}(\ulcorner L\urcorner) \wedge \neg \operatorname{Tr}(\ulcorner L\urcorner)$ (by A3). By (*), we obtain $L$ once again. Therefore, $T \vdash L$, and, so, $T \vdash B$, for every $B$ (by (*) and the fact that $T \vdash L \leftrightarrow \operatorname{Tr}(\ulcorner L\urcorner))$.

${ }^{24}$ It is also not immune to Curry's paradox.

${ }^{25}$ Notice that EC is a schema, expressing within the meta-language that for every predicate $P^{\prime}$ of the relevant theory, every instance of EC resulting from substituting $P$ by $P^{\prime}$ is an axiom of the theory. This means that EC does not fully internalize (the atomic-level version of) PNC-EC within the object-language - e.g., the way a formulation resorting to second-order quantification would do. I do not see this as a problem, though. For consider the analogous situation 
with respect to the principle of explosion: at the metatheoretic level this principle is usually formulated as stating that every pair of contradictory sentences entails any other sentence of the language. This formulation cannot be fully expressed within the object-language of many first-order theories - this would require, for example, quantifying over sentences and having a predicate standing for the entailment relation. Nonetheless, we still regard a theory whose theorems include all instances of the schema PGE as a theory countenancing the principle of explosion.

26 'PP' is an acronym for 'Parmenidean Principle'. Here is a sketch of the derivation of PP from EC in LFI1: by axiom A9, $\bullet E(v) \vee \circ E(v)$. Suppose $\bullet E(v)$. By A12 and A4, $E(v)$, and, so, $\circ E(v)$ (by EC, where $P$ is instantiated by $E$ ). Hence, $\bullet E(v) \vdash \circ E(v)$. Since $\circ E(v) \vdash \circ E(v)$, EC $\vdash \circ E(v)$ (by axioms A8 and A9).

${ }^{27}$ It might be suggested that $E$ could, in principle, be allowed to have contradictory instances. After all, although semantic dialetheism is compatible with the denial of ontological dialetheism, it is certainly compatible with it. A philosopher endorsing both semantic and ontological dialetheism could, of course, resort to an existence predicate in regimenting her theories, while still denying the Parmenidean Principle. However, since PP follows from EC, she will then have either to abandon EC or restrict it somehow. In any case, I do not think that such a philosopher would find EC attractive in the first place.

${ }^{28} \mathrm{~A}$ term $t$ is free in $A$ if either $t$ is closed or $t$ is a variable free in $A$.

${ }^{29}$ See footnote 26 for a qualification of this claim. 